\title{
Baroreceptor stimulation: Pain perception and sensory thresholds
}

\author{
Conrad Droste ${ }^{a}$, Attila Kardos ${ }^{a}$, Stuart Brody ${ }^{c}$, Mark W. Greenlee ${ }^{\text {, }}$, \\ Helmut Roskamm ", Harald Rau *.c \\ ${ }^{a}$ Benedikt-Kreutz Cardiovascular-Rehabilitation Center, Bad Krozingen, Germany \\ ${ }^{b}$ Neurological University Clinic, Department of Neurophysiology, University of Freiburg, \\ Freibury, Germany \\ "Institute for Medical Psychology and Behavioral Neurobiology, University of Tübingen, \\ Gartenstrasse 29,72074 Tübingen, Germany
}

(Received 21 April 1993; revised 16 July 1993; accepted 23 August 1993)

\begin{abstract}
Baroreceptor activity has been implicated in the modulation of pain. Sensory detection thresholds and pain ratings were measured in a group of 28 men during carotid baroreceptor manipulation with the PRES (phase-related external suction) neck suction technique. Brief, cardiac phase-related electrical impulses were delivered intracutaneously to the finger. The results indicate that minimum baroreceptor activity was associated with more severe pain, but had no effect on sensory detection threshold. The results are discussed in terms of the learned model of hypertension.
\end{abstract}

Keywords: baroreceptors; PRES; blood pressure; antinociception.

\section{Introduction}

A negative relationship between tonic blood pressure and pain sensitivity has been repeatedly observed in humans (Bruehl, Carlson \& McCubbin, 1992; Ghione, Rosa, Mezzasalma \& Panattoni, 1988; Ghione, Rosa, Panattoni, Nuti, Mezzasalma \& Giuliano, 1985; Maixner, 1991; Zamir \& Shuber, 1980) and animals (Maixner, Touw, Brody, Gebhart \& Long, 1982). One link between the cardiovascular system and the central nervous system is the arterial baroreceptors which serve as sensors for blood pressure and its changes and which are involved in cardiovascular homeostasis (Persson \& Kirchheim, 1991). Dworkin, Filewich, Miller, Craigmyle \& Pickering (1979) and Randich \& Maixner (1984) demonstrated that pharmacologically induced blood pressure increases in rats led to antinociception, but only if baroreceptors were left intact. Surgical denervation of baroreceptor afferents eliminated the antinociceptive effect of blood pressure increases.

* Corresponding author. 
In experimental investigations of the antinociceptive effects of baroreceptor activation in humans, baroreceptor activity is the independent variable. Pharmacological blood pressure manipulations (e.g. Larbig, Elbert, Rockstroh, Lutzenberger \& Birbaumer, 1985), change in body position (e.g. Vaitl \& Gruppe, 1992) or modification of the extramural pressure of the carotid sinus by means of a neck cuff (e.g. Toon, Bergel \& Johnston, 1984) to manipulate baroreceptor activity all share certain disadvantages. Specific baroreceptor effects cannot easily be differentiated from nonspecific effects (such as the direct action of blood pressure enhancing agents on the brain, distraction, aversiveness of cuff pressure changes, and speed and angle-dependent psychological perturbations caused by body tilting) which might have a direct influence on the pain processing system. Recently, Rau, Elbert, Geiger \& Lutzenberger (1992) developed a modified neck cuff technique called PRES (phase-related external suction) which allows for the noninvasive manipulation of the carotid baroreceptors without nonspecific artifacts. On a group level, subjects were not able to differentiate stimulation from a control condition (Furedy, Rau \& Roberts, 1992). By applying the PRES technique, Rau et al. (in press) found mechanical but not thermal pain was significantly reduced during baroreceptor stimulation. On the other hand, thermal but not mechanical pain was related to resting blood pressure with thermal pain sensitivity being reduced in subjects with higher resting blood pressure. Thermal and mechanical pain models do not lend themselves to the brief time periods necessary for synchrony with specific cardiac phases. However, electrical stimuli can be extremely brief and precisely timed, and were chosen for the current study to examine the interactive effect of baroreceptor manipulation and cardiac cycle on pain and sensory threshold.

The present investigation had two purposes: First, to investigate whether PRES reduces electrically elicited pain (which operates differently from thermal or mechanical pain) and secondly, to investigate whether PRES baroreceptor manipulation is specific in its antinociceptive effect, or if it works by through a global diminution of sensation, as might be hypothesized from the sedative effect of baroreceptor stimulation on Koch's dogs (Koch, 1937) or the finding of anticonvulsive effects of baroreceptor activation in animals (Gellhorn, Yesinick, Kessler \& Hailman, 1942). The second issue is examined in the present study by evaluation of sensory detection thresholds during various levels of baroreceptor activity.

\section{Method}

\section{Subjects}

Twenty-eight subjects, aged 21-67, participated in the study. The exclusion criteria for the study were: (1) being over age 70; (2) illness other than 
hypertension; (3) arrhythmias or conduction disturbances in the ECG; (4) carotid-sinus hyperesthesia (measured during ECG recording), stenosis of the common and internal carotid arteries and carotid artery flow abnormalities as determined by two dimensional and color Doppler echocardiography (Aloka 720); or (5) coronary heart disease. Subjects were asked to forego all medication for $48 \mathrm{~h}$ prior to the investigation. They were also requested to refrain from alcoholic beverages for $24 \mathrm{~h}$ before the study, as well as caffeinated beverages and smoking on the day of the examination. Subjects were tested while seated upright in a dental chair in a quiet room. After arrival in the laboratory, blood pressure was measured with a sphygmomanometer.

\section{Ethical approval}

The study was approved by the University of Tübingen ethics committce. At the time of examination, the subjects were given a written explanation of the general research aims and possible hazards. At this time they were asked to sign a statement of informed consent. Emphasis was placed on the voluntary nature of participation, as well as on the possibility of discontinuing participation at any time. The subjects were informed that the results of the investigation would be kept strictly confidential.

\section{Physiological recordings}

The electrocardiogram was measured using Beckman $\mathrm{Ag} / \mathrm{AgCl}$ electrodes which were attached to the thorax at positions producing clear R-waves. The $\mathrm{R}$-wave of the electrocardiogram was detected by a cardiotachometer and the resulting signal was transmitted to a digital input channel of an input-output board (Data Translation 2821) in an IBM-AT compatible computer. The computer measured the interval between successive $\mathrm{R}$-waves with a precision of $0.5 \mathrm{~ms}$. The inter-beat intervals were calculated off line, and transformed into heart rate.

\section{PRES baroreceptor manipulation}

The baroreceptors are stretch receptors sensitive to changes in transmural pressure normally induced by transient increments and decrements in intravascular pressure (Mancia, 1983; Mancia, Ludbrook, Ferrari, Gregorini \& Zanchetti, 1978). Phasic increases in blood pressure stimulate the baroreceptors to decrease heart rate (the baroreflex). The baroreceptors can also be stimulated by changes in extravascular pressure (e.g. neck cuff pressure (Mancia et al., 1977)), as well as by the combination of extravascular and intravascular pressures. 
Baroreceptor manipulation was conducted according to the PRES method described by Rau and coworkers (1992). A malleable neck cuff was positioned on the subject's neck and secured with an elastic band and Velcro tape. PRES neck-suction was implemented by computer-controlled valves which directed motor-driven air, and which were synchronized to the heart cycle. Changes in local cuff pressure from -30 to $+10 \mathrm{mmHg}$ could be achieved within $180 \mathrm{~ms}$ of valve operation.

The four PRES conditions are schematically illustrated in Fig. 1. They were systolic suction/diastolic blowing with the pain stimulus delivered during either systole (1) or diastole (2), and diastolic suction/systolic blowing, with pain delivered during either systole (3) or diastole (4). During systole, the highest level of endogenous baroreceptor activity occurs, while such activity is low during diastole. These natural fluctuations can be augmented by PRES, which increases firing of the baroreceptors through suction, and decreases such firing by blowing. Superimposition of PRES suction on systole maximizes vascular dilation and thus results in the highest levels of baroreceptor activity, while the combination of positive pressure and diastole decreases blood vessel diameter and results in the lowest rate of baroreceptor firing.

In the systolic suction conditions, a pulse of negative pressure (suction) coincided with systole and the passing of the pulse wave through the internal carotis, whereas a positive pressure pulse (blowing) was delivered during diastole.

In the diastolic suction conditions, a positive pressure pulse was delivered during systole and negative pressure was produced in the neck cuff during diastole. In all PRES conditions, pressure pulses commenced $100 \mathrm{~ms}$ following detection of the cardiac $\mathrm{R}$-wave, and were equal in duration to one-half of the previously recorded mean interbeat interval minus $100 \mathrm{~ms}$. The diastolic pressure pulse directly followed the systolic pulse, and the pulses were of equal duration. This sequence was repeated for $6 \mathrm{~s}$ in each trial. The conditions were presented in a pseudo-randomized order.

\section{Sensory detection thresholds and electrical pain stimuli}

The experiment was conducted in two parts on separate days. In most cases these were consecutive days, and in the other cases they were separated by one additional day. The experiments were performed at the same time of day for each subject. During day 1, the influence of baroreceptors on perception threshold was investigated, and during day 2 the influence on pain was examined.

The electrical stimuli were generated by a specially designed optically isolated, constant current, stimulus generator. This device produced electrical impulses, the current amplitude of which was defined as a Gaussian 

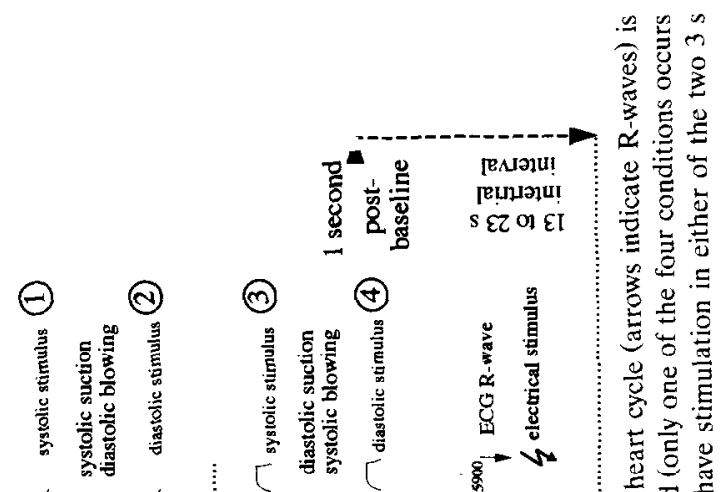

$\oplus$
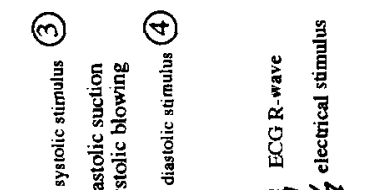

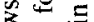

\% 过若 을 它 졸

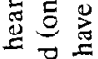
导总 造苟

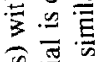

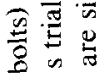
an 0 政 要 然 5 影 实 冚. क 콩크믕 E 过苛 (0. E. on 两告 है 옹 零 로롤 음 寻 壳点。 0 昰 岂 \& 昰造 $\infty=$ 灵豆 . 한 - $=2$

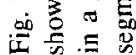


function of time. The stimulus duration was $10 \mathrm{~ms}$. These electrical impulses were applied via an intracutaneous, stainless steel electrode (diameter, $1 \mathrm{~mm}$; length, $1.5 \mathrm{~mm}$ ) to the tip of the left middle finger (Bromm \& Maier, 1984). To reduce cutaneous resistance and uniformly lower sensory thresholds, the superficial layers of the epidermis of the middle finger of the left hand were removed with a hand-held dental burr (diameter, $1.5 \mathrm{~mm}$ ). Care was taken to avoid damage to the underlying corium. A reference electrode was fastened to the flexor region of the left forearm.

Sensory detection thresholds and pain ratings were determined in each of the four experimental conditions, which were presented in individually created pseudo-random order. The beginning and end of each trial was announced by computer-generated tones.

A few practice trials were run to ensure that the electrodes were properly positioned and that the PRES and fingertip equipment were functioning. At this time, the subject was familiarized with the action of the neck cuff, the sounds indicating the onset of a trial, finger stimulation and rating procedures.

\section{Sensory threshold determination}

Each trial was divided into two intervals of $3 \mathrm{~s}$ each. The computer algorithm selected on a pseudo-random basis whether the stimulus was delivered in the first or second interval. The stimulus was delivered in the latter half of the $3 \mathrm{~s}$ interval so chosen. The exact onset of the stimulus was phase locked in time to the cardiac cycle, either $200 \mathrm{~ms}$ after ECG R-wave detection in the case of systolic stimulus presentation, or $100 \mathrm{~ms}$ after cuff pressure reversal in the case of diastolic stimulus presentation (see Fig. 1). Subjects indicated with a forced-choice button press the interval in which they believed the stimulus was delivered. A staircase procedure was employed to determine the stimulus amplitude at which the subject responded correctly in $75 \%$ of the trials for each condition. Subjects typically required about 80 trials. The stimulus amplitude varied from a value well below detection threshold $(0.02 \mathrm{~mA})$ to $0.5 \mathrm{~mA}$ in 40 equal intensity steps. The first trial began with the highest value possible and for each subsequent trial the stimulus amplitude which had the greatest likelihood of corresponding to the actual threshold value was chosen, according to an adaptive algorithm (the so-called Best PEST: parametric estimation by sequential testing) method described by Lieberman \& Pentland (1982).

\section{Pain ratings}

The pain stimulus was five times the sensory detection level, but intermediate values (integer multiples of the previously determined sensory detection 
threshold) were also delivered. Before the 164 experimental trials ( 30 of each of the five intensities plus 14 "catch" trials presented in pseudorandomized order), the full pain stimulus was presented as a reference. Subjects were given a linear potentiometer, which could be adjusted by moving a dial along a $10 \mathrm{~cm}$ scale. A marker was positioned $8 \mathrm{~cm}$ above the lowest setting (the lowest setting corresponding to zero magnitude) and $2 \mathrm{~cm}$ below the highest setting (the highest setting corresponding to maximum or 1.0 magnitude), and subjects were told that the reference stimulus corresponded to that $8 \mathrm{~cm}$ point. Subjects were instructed to use this device to rate the painfulness of the stimuli, following each trial. The experimental instructions gave no indication that only five amplitude levels would be used in the experiment, nor was there any mention of "catch trials" (trials containing no stimulus). They were informed that in trials in which they were unable to detect the stimulus the zero magnitude level should be selected. After the subject was certain of the rating, the subject pressed a button causing the computer to record the response and proceed to the next trial.

\section{Data analysis}

Data acquisition, control of experimental stimuli, and PRES operation were implemented by a computer program written in ASYST (KeithleyMetrabyte, Rochester, NY). Heart rate responses and sensory thresholds were averaged separately for the four experimental conditions. Pain ratings of the highest stimulus intensity were also averaged for the four experimental conditions. The remaining stimulus intensity levels were included in the experiment to provide variability of stimuli, but not in the analysis as we were only interested in the effect of baroreceptor manipulation on stimuli which were clearly painful to all subjects (as verified by the verbal reports of the subjects). Separate analyses of variance (ANOVA) were calculated to test the effects of the repeated measures within-subject factor PRES condition (systolic negative pressure vs. diastolic negative pressure with stimuli delivered during either systole or diastole) on sensory detection threshold, heart rate and pain rating. Significant interactions were followed up with post hoc comparisons. Pearson correlation coefficients were computed for ancillary investigations including the interrelationship of resting blood pressure, age, baroreceptor sensitivity (heart rate change) and pain rating.

\section{Results}

Effect of baroreceptor stimulation on heart rate

The PRES conditions had significantly different effects on heart rate responses $(F(1,26)=70.0 ; p<.0001)$. The baroreceptor stimulation condi- 
Table 1

Sensory detection thresholds

$\begin{array}{lllll}\text { PRES condition } & \text { Stimulus } & \text { Condition } & \begin{array}{l}\text { Mean } \\ \text { detection } \\ \text { threshold } \\ (\mathrm{mA})\end{array} & \begin{array}{l}\text { Standard } \\ \text { deviation }\end{array} \\ \text { Systolic suction } & \begin{array}{l}\text { Systole } \\ \text { Diastole }\end{array} & 1 & 0.165 & 0.086 \\ & 2 & 0.162 & 0.061 \\ \text { Diastolic suction } & \text { Systole } & 3 & 0.175 & 0.093 \\ & \text { Diastole } & 4 & 0.169 & 0.074\end{array}$

tion (systolic suction) evoked larger heart rate decelerations $(3.3 \mathrm{bpm})$ than diastolic suction trials $(0.6 \mathrm{bpm})$, averaged for pain stimulus timing within the heart cycle.

\section{Sensory detection thresholds}

Table 1 presents the sensory detection thresholds under each of the four PRES conditions. There was no baroreceptor effect on detection threshold $(F(3,84)=0.34, p=.80)$, as depicted in Fig. 2 .

\section{Pain ratings}

Table 2 presents the pain ratings for each of the four PRES conditions. As depicted in Fig. 2, the experimental intervention had a significant effect on pain rating $(F(3,81)=4.11, p=.009)$, with post hocs indicating the most
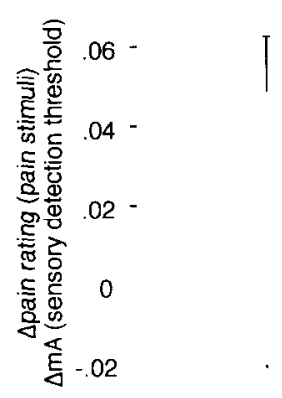

pain stimuli sensory detection

Fig. 2. Means and standard errors of difterential pain ratings (left) and sensory detection thresholds (right, $\mathrm{mA}$ ) between systolic suction/diastolic blowing PRES conditions with diastolic and systolic stimuli, the conditions with minimum and maximum baroreceptor activity. 
Table 2

Pain ratings

PRES condition

Stimulus

Condition

Mean

Standard

pain deviation

Systolic suction

Systole

rating

Diastolic suction

Diastole

0.622

0.176

0.670

0.169

Systole

0.634

0.160

Diastole

0.628

0.154

severe pain rating during minimum baroreceptor activity (diastolic blowing, diastolic pain delivery) compared to all other conditions $(t=3.33, p=.003)$. No other significant differences between a given condition and the mean of all others was found.

\section{Ancillary investigations}

As a measure of individual baroreceptor sensitivity, we calculated the difference between heart rate change in the systolic $6 \mathrm{~s}$ of baroreceptor activation per trial) and diastolic suction (control) conditions. This measure had a mean of -2.7 , and a standard deviation of 1.96 . This differential response was related to both age $(r=-.66, p<.001)$, and diastolic blood pressure $(r=-.50, p=.006)$, with greater age or blood pressure being associated with less baroreceptor sensitivity. However, in our sample, age was positively correlated with blood pressure (systolic $r=.61, p<.001$; diastolic $r=.68, p<.001$ ), and partialling out the effect of age from the correlation between baroreceptor sensitivity and both systolic and diastolic resting blood pressure left no significant correlations (all $|r \mathrm{~s}|<.2$ ). Hence, the ultimate source of this effect is unclear.

This differential heart rate index of baroreceptor sensitivity was unrelated to change in pain ratings $(|r|<.1)$. Change in pain ratings was also unrelated to age $(|r|<.2)$ and both diastolic $(r=.25)$ and systolic $(r=.24)$ blood pressure.

Heart rate (both resting and during each of the four PRES conditions) was unrelated to diastolic and systolic blood pressure, age, and baroreceptor sensitivity (all $|r \mathrm{~s}|<.2$ ).

\section{Discussion}

The heart rate responses to neck-cuff stimulation indicate that the PRES procedure had a reliable effect on baroreceptor activity, albeit smaller in 
magnitude than that obtained with uncontrolled methods. Our previous research has demonstrated reliable PRES effects on blood pressure and heart rate (Rau, Brody, Droste \& Kardos, 1993). In addition to these cardiovascular effects, extrahomeostatic effects including diminution of cortical activity (Rau, Pauli, Brody, Elbert \& Birbaumer, 1993; Elbert et al., 1988), spinal reflex amplitude (Rau, Brody, Brunia, Damen \& Elbert, in press), and mechanical pain verify the usefulness of PRES in studies of the effect of baroreceptor manipulation on psychological and physiological processes.

Because sensory threshold procedures were conducted prior to pain ratings (on separate days), it is possible that a habituation or familiarization effect may have occurred, leading to lower intensity estimates. However, these sensory threshold values were essential for determining the pain stimuli values, and thus the sequence could not be counterbalanced. In any event, the comparison of baroreceptor conditions would not be affected by this lack of counterbalancing.

Artificially disrupting baroreceptor activity by means of PRES led to more severe electrical pain ratings, consistent with earlier results from our laboratory (Rau, Elbert, Pauli \& Birbaumer, 1993). I lowever, none of the baroreceptor manipulations had an effect on sensory thresholds (with adequate statistical power), implying that baroreceptor activity is specifically antinociceptive and does not dull sensation of non-painful stimuli. Earlier findings of decreased central nervous system arousal as indexed by reduction of the contingent negative variation of the EEG (CNV, Rau et al., 1993; Elbert et al., 1988) may be in accord with the present result. The CNV studies involved delivery of electrical pain stimuli, and $\mathrm{CNV}$ reduction may therefore reflect diminished attention towards the aversive experience. While the CNV studies reflected non-specific decrements of CNS arousal processes (Birbaumer, Elbert, Canavan \& Rockstroh, 1990), the present study implies a specific reduction in pain, although it is possible that a more intense form of baroreceptor stimulation would produce a more global reduction of consciousness.

The finding of decreased baroreceptor sensitivity with increasing age and tonic blood pressure is consistent with the literature (Gribbin, Pickering, Sleight \& Peto, 1971; Korner, West, Shaw \& Uther, 1974; Randall et al, 1976; Randall, Esler, Culp, Julius \& Zweifler, 1978). In the present study, the heart rate change measure of baroreceptor sensitivity was unrelated to the differential effect of baroreceptor activity on pain ratings, implying homeostatic and extrahomeostatic effects of baroreceptor activity may have individual sensitivities. For example, CNV changes were negatively related to heart rate changes in the Elbert, Tafil-Klawe, Rau \& Lutzenberger (1991) study.

Possible clinical implications of the present results include support for the operant development of hypertension as proposed by Dworkin et al. (1979). It was proposed that the negatively reinforcing properties of phasic increases 
in blood pressure result in the development of hypertension in susceptible individuals. Susceptibility would be a joint function of capacity for blood pressure increases, a reinforcing effect of such increases, and chronic stressful or painful experience. That model was based on animal studies with pharmacological manipulation of blood pressure. Elbert et al. (1988) extended the findings to humans with the (uncontrolled) neck-cuff technique. The present study clarifies that controlled low intensity baroreceptor stimulation (in contrast to the high intensity stimulation used by Koch, 1937) decreases pain but not sensitivity to non-aversive stimulation. The next step in evaluating the learned hypertension hypothesis (already under way in the Tübingen laboratory) is a longitudinal examination of the relation between baroreceptor-dependent antinociception and the development of higher blood pressure.

\section{Acknowledgements}

This research was supported by the Deutsche Forschungsgemeinschaft (SPP II-B-7-Dr 190-2 to C. Droste and BI 195/24 to H. Rau).

\section{References}

Birbaumer, N., Elbert, T., Canavan, A., \& Rockstroh, B. (1990). Slow potentials of the cerebral cortex and behavior. Physiological Reviews, 70, 1-41.

Bromm, B., \& Maier, W. (1984). The intracutaneous stimulus; a new pain model for algesimetric studies. Methods and Findings of Experimental and Clinical Pharmacology, 6, 405-410.

Bruehl, S., Carlson, C.R., \& McCubbin, J.A. (1992). The relationship between pain sensitivity and blood pressure in normotensives. Pain, 48, 463-467.

Dworkin, B., Filewich, R.J., Miller, N.E., Craigmyle, N., \& Pickering, T.G. (1979). Baroreceptor activation reduces reactivity to noxious stimulation: Implications for hypertension. Science, $205,1299-1301$.

Elbert, T., Lutzenberger, W., Rockstroh, B., Kessler, M., Pietrowsky, R., \& Birbaumer, N. (1988). Baroreceptor stimulation increases pain threshold in borderline hypertensives. Psychophysiology, 25, 25-29.

Elbert, T., Tafil-Klawe, M., Rau, H., \& Lutzenberger, W. (1991). Cerebral and cardiac responses to unilateral stimulation of carotid sinus baroreceptors. Journal of Psychophysiology, 5, $327-335$.

Furedy, J., Rau, H., \& Roberts, L. (1992). Physiological and psychological differentiation of bidirectional baroreceptor carotid manipulation in humans. Physiology and Behavior, 52, 953-958.

Gellhorn, E., Yesinick, L., Kessler, M., \& Hailman, H. (1942). Carotid sinus reflexes and convulsions. American Journal of Physiology, 137, 396-403.

Ghione, S., Rosa, C., Mezzasalma, L., \& Panattoni, E. (1988). Arterial hypertension is associated with hypalgesia in humans. Hypertension, 12, 491-497. 
Ghione, S., Rosa, C., Panattoni, E., Nuti, M., Mezzasalma, L., \& Giuliano, G. (1985). Comparison of sensory and pain threshold in tooth pulp stimulation in normotensive men and essential hypertension. Journal of Hypertension, 3 (suppl 3), S113-S115.

Gribbin, B., Pickering, T.G., Sleight, P., \& Peto, R. (1971). Effect of age and high blood pressure on baroreflex sensitivity in man. Circulation Research, 29, 424-431.

Koch, E.B. (1937). Die Irradiation der pressoreceptorischen Kreislaufreflexe. In A. Schweitzer (Ed.), Die Irridation autonomer Reflexe. Basel: Karger.

Korner, P.I., West, M.J., Shaw, J., \& Uther, J.B. (1974). "Steady-state" properties of the baroreceptor-heart rate reflex in essential hypertension in man. Clinical and Experimental Pharmacological Physiology, 1, 6576.

Larbig, W., Elbert, T., Rockstroh, B., Lutzenberger, W., \& Birbaumer, N. (1985). Elevated blood pressure and reduction of pain sensitivity. In J.F. Orlebeke Mulder, G., van Doornen, J.P. (Eds.), Psychophysiology of cardiovascular control. (pp. 113-122). New York: Plenum.

Lieberman, H.R., \& Pentland, A.P. (1982). Microcomputer-based estimation of psychophysical thresholds: The Best PEST. Behavior Research Methods \& Instrumentation, 14, 21-25.

Maixner, W. (1991). Interactions between cardiovascular and pain modulatory systems: physiological and pathophysiological implications. Journal of Cardiovascular Electrophysiology, 2, S3-S12.

Maixner, W., Touw, K.B., Brody, M., Gebhart, G.F., \& Long, J.P. (1982). Factors influencing the altered pain perception in the spontaneously hypertensive rat. Brain Research, 237, 137-145.

Mancia, G. (1983). Reflex control of circulation in normotensive and hypertensive humans. In D. Ganten \& D. Pfaff (Eds.), Central cardiovascular control (pp. 63-86). Berlin: Springer-Verlag.

Mancia, G., Ferrari, A., Gregorini, L., Valentini, R., Ludbrook, J., \& Zanchetti, A. (1977). Circulatory reflexes from carotid and extracarotid baroreceptor areas in man. Circulation Reseurch, 41, 309-315.

Mancia, G., Ludbrook, J., Ferrari, A., Gregorini, L., \& Zanchetti, A. (1978). Baroreceptor reflexes in human hypertension. Circulation Research, 43, 170-177.

Persson, P.B., \& Kirchheim, H.R. (Eds.). (1991). Baroreceptor reflexes. Berlin: Springer-Verlag.

Randall, O.S., Esler, M.D., Bulloch, G.F., Maisel, A.S., Ellis, C.N., Zweifler, A.J., \& Julius, S. (1976). Relationship of age and blood pressure to baroreflex sensitivity and arterial compliance in man. Clinical Science of Molecular Medicine Supplement, 3, 357S-360S.

Randall, O.S., Esler, M.D., Culp, B., Julius, S., \& Zweifler, A.J. (1978). Deteminants of baroreflex sensitivity in man. Journal of Laboratory and Clinical Medicine, 91, 514-519.

Randich, A., \& Maixner, W. (1984). Interactions between cardiovascular and pain regulatory systems. Neuroscience \& Biobehavioral Reviews, 8(3), 343-367.

Rau, H., Brody, S., Brunia, C.H.M., Damen, E.P.J., \& Elbert, T. (in press). Activation of carotid baroreceptors inhibits spinal reflexes in man. Electroencephalography and Clinical Neurophysiology.

Rau, H., Brody, S., Droste, C., \& Kardos, A. (1993). Blood pressure changes validate phase-related external suction, a controlled method for stimulation of human baroreceptors. European Journal of Applied Physiology, 67, 26-29.

Rau, H., Brody, S., Larbig, W., Pauli, P., Vöhringer, M., Harsch, B., Kröling, P.. \& Birbaumer, $\mathrm{N}$. (in press). The effects of PRES baroreceptor stimulation on thermal and mechanical pain threshold in borderline hypertensives and normotensives. Psychophysiology.

Rau, H., Elbert, T., Geiger, B., \& Lutzenberger, W. (1992). PRES: The controlled noninvasive stimulation of the carotid baroreceptors in humans. Psychophysiology, 29(2), 165-172.

Rau, H., Elbert, T., Pauli, P., \& Birbaumer, N. (1993). Bluthochdruck eine gelernte Reaktion? Implikationen eines systemtheoretischen Modells. Verhaltensmodifikation und Verhaltensmedizin, 14, 96-120.

Rau, H., Pauli, P., Brody, S., Elbert, T., \& Birbaumer, N. (1993). Baroreceptor stimulation alters cortical activity. Psychophysiology, 30, 322-325. 
Toon, P.D., Bergel, D.H., \& Johnston, D.W. (1984). The effect of modification of baroreceptor activity on reaction time. Psychophysiology, 21, 486-494.

Vaitl, D., \& Gruppe, H. (1992). Body position and changes in EEG. Journal of Psychophysiology, $6,111-118$.

Zamir, N., \& Shuber, E. (1980). Altered pain perception in hypertensive humans. Brain Research, 201, 471-474. 